\title{
PITCHING STABILITY SIMULATION OF A BIONIC COWNOSE RAY
}

\author{
Yueri Cai, Jun Gao and Shusheng Bi \\ Robotics Institute, Beihang University, \\ Beijing 100191, P.R. China \\ E-mail: biss_buaa@163.com
}

\author{
Cong Liu and Houxiang Zhang \\ Aalesund University College, \\ Aalesund 6025, Norway \\ E-mail: hozh@hials.no
}

\author{
KEYWORDS \\ Bionic fish, Pitching stability, Simulation
}

\begin{abstract}
Swimming stability is essential to a bionic robotic fish which is aiming to be applied to practical application. The stability can be controlled through two kinds of methods, the passive control method and the active control method. The latter one performs more flexibility. Forces of disturbance caused by movements of the bionic pectoral foils and the horizontal tail of a bionic fish imitating cownose ray propelled by oscillating paired pectoral foils are analyzed. Simulation model based on both PID method and fuzzy control method for the stability performance of the bionic fish in condition of compensating work by horizontal tail or not are built. Results show that the stability of the bionic fish can be obviously improved by actively control of the horizontal tail.
\end{abstract}

\section{INTRODUCTION}

Fish propelled by oscillating paired pectoral foils occupies excellent stability and maneuverability (Harris 1938; Rosenberger 2001; Suzuki et al. 2007; Webb 2002). Cownose ray is a typical fish occupying these features (Parson 2011). Some researchers and engineers have been trying to understand the swimming mechanics and to explore the bio-inspired methodology in the design and development of bionic underwater vehicles mimicking this kind of fish (Cai et al. 2010 and 2012; Chen et al. 2011; Elizabeth 2011; IMAE 2012; Wang et al. 2009; Xu et al. 2007; Zhou and Low 2010). As for applications of bionic robotic fish, especially for underwater detection and observation, the ability of stability performs an essential role (Anderson and Chhabra 2002; Hu et al. 2006; Sefati et al. 2012; Wang et al. 2005). Some extent of additional forces will be generated by the oscillating fins when they generate effectual propulsion force by interacting with the coming flow. However, the additional forces have negative effects on swimming stability of bionic robotic fish as they usually pointing to other directions than the useful driving force.

Swimming stability of the robotic fish is essential to the effective propulsion force generation from bionic flapping foils. The swimming speed of a bionic fish propelled by tail fins will be reduced to only $1 / 3$ of the normal, on condition that the yawing motion is not controlled properly, for the movement of flapping fins is weakened and distortion (Wang et al. 2005). The movement mode of fish with paired pectoral foils is different from the ones propelled by tail fins, which can provide better stability. Distance between the gravity center and the propulsion part of the bionic fish utilizing $\mathrm{BCF}$ type is large compared with its body length. An obvious yaw motion will be suffered by only small lateral force usually. As for the propulsion parts of bionic fish utilizing MPF type, oscillating paired pectoral foils, are much closer to the gravity center comparatively. Therefore, the additional force generated has limited impact on the stability performance. But, for the same kind of bionic fish mimicking cownose ray, some research have attained the results that the pitching angle must be strictly controlled to within a small range, otherwise the hydrodynamic resistance will increase dramatically and the propulsion efficiency will be reduced (Wang et al. 2005). As a whole, the stability of a bionic fish is essential to its desired performances with high efficiency and high speed as its biomimetic samples in nature. The stability can be improved through optimization of mechanical design and making effective utilization of assisted movement of other functional parts of bionic prototypes developed. Importance of the stability is realized by researchers focusing on studying bionic robotic fish and some research have been carried out either by simulation or experiments (Wilson and Eldredge 2011; Xu et al. 2012; Yu et al. 2012).

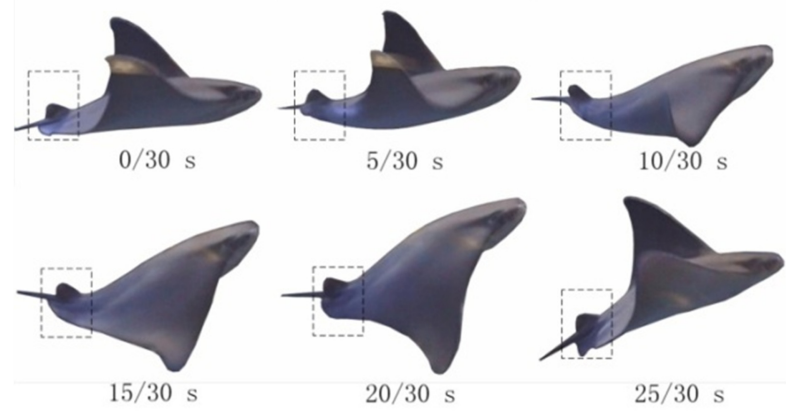

Figures 1: Up-floating Movement of Cownose Ray Assisted by Tail

Based on observations of live cownose ray in aquarium, besides the flexible flapping movement of its pectoral foils, obvious swing motion of the tail part of cownose ray is utilized to support the up-floating and diving movement. Direction of its head during straight forward swimming can be adjusted partly by the supporting movement of its tail. Snapshots of up-floating movement of a sample cownose ray are shown in Fig. 1. During the stable straight forward swimming, the swing 
motions with low-amplitude of its tail are used to keep its body with stability. Effect of the tail part of cownose ray is similar with combined work of rudder and elevator of fix-wing aircraft. Therefore, the simulation and control of the bionic fish propelled by oscillating paired pectoral foils discussed in this paper are realized by a flat elevator mounted on the tail part of the bionic fish.

There are two kinds of methods to control stability of the bionic fish: one is natural stability or static stability, the other is controlled stability (Sun 2011). The underwater vehicle can recover to stable state when suffered by external interference, if it occupies ability of static stability. In condition that the underwater does not occupy this ability or the static stability does not work well, the method of controlled stability should be added to enhance the stability performance. Requirements of design will be reduced by utilizing method of controlled stability.

\section{BASIC SETTINGS}

The coordinate system set as shown in Fig. 2 is utilized in calculation and constructing the simulation model. The original point is set at the gravity center of the body of the bionic fish, $x$-axis is coincident with the central body axis and point to the head, $y$-axis is perpendicular to $x$-axis in middle horizontal plane and pointing to right and $z$-axis is decided by the law of Cartesian coordinate system.

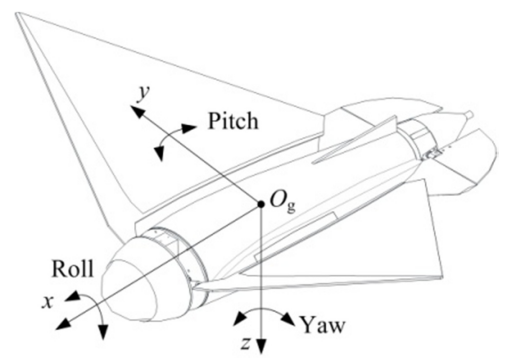

Figures 2: Coordinate System of the Bionic Fish

The settings shown in Fig. 3 are utilized here to analyze the pitching movement caused by the flapping pectoral foils and the horizontal tail of the bionic fish. When the angle generated is minus or the elevator swing downward, the torque generated will make the head of the bionic fish lower or diving; otherwise the angle is positive, the torque generated will make the head of the bionic fish higher or up-floating.

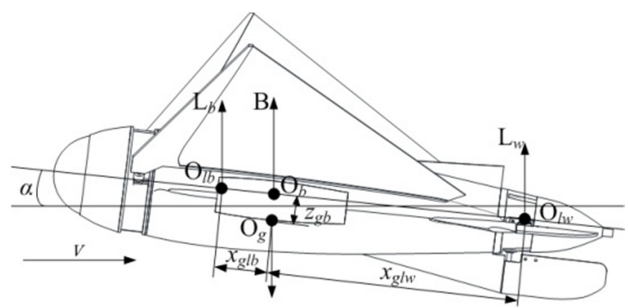

Figures 3: Force Diagram of the Bionic Fish Swimming with a Pitching Angle.

\section{DISTURBANCE BY PECTORAL FOIL}

To estimate the disturbance caused by the movement of the flapping foil qualitatively, the calculations of the disturbance force and torque are simplified here. The disturbance torque is mainly caused by the inertial force and fluid force caused by the flapping movement of the pectoral foils. Supposing the pectoral foils flap according to the discipline of:

$$
\begin{gathered}
\emptyset(t)=\emptyset_{0} \cos \omega t, \\
\dot{\emptyset}(t)=-\emptyset_{0} \omega \sin \omega t, \\
\ddot{\emptyset}(\mathrm{t})=-\emptyset_{0} \omega^{2} \cos \omega t .
\end{gathered}
$$

where $\varnothing$ is the flapping angle of the pectoral foil, $\emptyset_{0}$ is amplitude of the flapping angle and $\omega$ is the angle velocity.

Then, the inertial force generated by a finite element on the pectoral foil can be calculated as:

$$
d F_{f e}=-\rho_{f} \ddot{\emptyset} y d x d y .
$$

Resolution of the inertial force along $y$-axis and $z$-axis is:

$$
\begin{aligned}
& d F_{f e z}=-\rho_{f} \ddot{\emptyset} y \cos \emptyset d x d y, \\
& d F_{f e y}=-\rho_{f} \ddot{\emptyset} y \sin \emptyset d x d y .
\end{aligned}
$$

As the two pectoral foils are flap symmetrically, components of the force and torque along $y$-axis generated by the two pectoral foils are with equal value but opposite direction. The overall inertial torque generated by one pectoral foil is:

$$
M_{f e}=-\iint \rho_{f} \ddot{\emptyset} \cos \emptyset x y d x d y,
$$

where $\iint x y d x d y$ is product of inertia about $x$-axis and $y$-axis.

The lift generated by the flapping foils is consistent with sinusoidal function as the sinusoidal motion employed by the flapping pectoral foil, as:

$$
F_{f l}=F_{\max } \sin \omega t,
$$

where $F_{\max }$ is the maximum value of the lift force, and is defined by the frequency, amplitude, velocity of incoming flow and flexibility of the pectoral foil.

Then the pitching moment by a single pectoral foil is:

$$
M_{f l}=F_{f l} \cdot x_{f l}
$$

where $x_{f l}$ is the distance between the lift force application point and the $y$-axis. As the two pectoral foils flaps symmetrically, the overall disturbance moment can be calculated by: 


$$
M_{f}=\sqrt{k_{f 1}^{2}+k_{f 2}^{2}} \cdot \sin (\omega t+\varphi),
$$

where: $\quad k_{f 1}=2 \iint x y d x d y \rho_{f} \emptyset_{0} \omega^{2} \cos \emptyset$,

$$
\begin{gathered}
k_{f 2}=2 F_{\max } \cdot x_{f l}, \\
\varphi=\tan ^{-1}\left(\frac{k_{f 1}}{k_{f 2}}\right) .
\end{gathered}
$$

Then the differential equation of the pitching disturbance is

$$
\begin{gathered}
\left.\left(J_{\mathrm{b}}+\Delta \mathrm{J}_{\mathrm{b}}\right) \ddot{\alpha}+k_{w 2} \dot{\alpha}+\left(k_{b 1}-k_{b 2}+k_{w 1}\right) \alpha\right) \\
=A_{0} \cdot \sin (\omega t+\varphi),
\end{gathered}
$$

where $A_{0}=\sqrt{k_{f 1}^{2}+k_{f 2}^{2}}$ is the maximum value of the disturbance moment. Therefore, the pitching disturbance can be taken as vibration of a second-order damping system under harmonic excitation. The steady-state response of the second-order damping system is considered mainly in the simulation here.

The pitching disturbance is mainly affected by two factors: (1) the disturbance moment generated by the flapping pectoral foil. The frequency of the disturbance moment will be consistent with the flapping frequency and the amplitude of the disturbance moment will increase with the flapping frequency and amplitude. (2) the natural characteristics of the pitching system. Based on the analysis presented, the pitching disturbance movement can be controlled by minimized the moment generated by the flapping motion of the pectoral foil.

The method can be obtained by applying the following methods: (1) Utilizing materials featured with less density and more flexibility to lower inertial force and further lower the disturbance (Wilson and Eldredge 2011). (2) Reducing the frequency and amplitude of the flapping motion, which will make the swimming velocity be reduced too. (3) Improving the structure of the bionic fish to reduce the distance between the lift application point and the center of gravity, which is mainly relied on the realization of practical swimming function. (4) Changing the natural characteristics of the pitching movement system of the bionic fish, including the equivalent moment inertia, the equivalent damping and equivalent rigidity. Although these methods can lower the pitching disturbance, realizing these methods in practical development of a real bionic fish is complicated and difficult. Therefore, a better method to improve stable ability is to make use of additional parts, such as horizontal tail mounted on the tail part of the bionic fish, to auxiliary control the pitching disturbance timely.

\section{DISTURBANCE BY HORIZONTAL TAIL}

As shown in Fig. 2, supposing the pitching angle is $\alpha$ during straight forward swimming of the bionic fish, torques relative to the gravity center will be generated by the body and the horizontal tail in this condition. Both torques generated by the body and the tail are analyzed through a same method. The flow around the tail will be infected by the body. Under action of viscous effect of the body, the speed of the flow over body will be lower. Generally, the velocity coefficient is set as $k$, so the flow velocity at the tail part is (Fang 2005):

$$
V_{w}=\sqrt{k} V .
$$

Considering the disturbance pitching angle is actually small and swimming velocity of the bionic fish we developed propelled by oscillating paired pectoral fins is much lower than its bionic sample, about 0.7 times of its body length per second, the induction effect on the flow direction is omitted here. The horizontal tail is put on the middle section of the tail part of the body, in condition of zero lift generation the attack angle will be zero too:

$$
\alpha_{0 w}=0
$$

The lift generated by the flat tail will be

$$
L_{\mathrm{w}}=0.5 C_{\mathrm{w}} \rho V_{\mathrm{w}}^{2} S_{\mathrm{w}} \alpha,
$$

where $C_{\mathrm{w}}$ is the lifting line slope of the horizontal tail, $S_{\mathrm{w}}$ is the effective cross-section area of the flat tail. As shown in Fig. 3, supposing the application point of the force generated by the flat tail is $O_{\mathrm{lw}}$, and the distance between the application point and the gravity center is $x_{\mathrm{glw}}$, then the pitching torque will be

$$
\mathrm{M}_{\mathrm{lw}}=-\left(0.5 C_{\mathrm{w}} \rho k V^{2} S_{\mathrm{w}} x_{\mathrm{glw}} \cos \alpha\right) \alpha .
$$

During the swing motion, resistance of the horizontal tail can be analyzed as follows. Set $\dot{\alpha}$ as the rotational angle velocity, then the additional velocity at the force application point $O_{\mathrm{lw}}$ is

$$
\Delta V=\dot{\alpha} x_{\mathrm{glw}}
$$

The variation of average attack angle of the horizontal tail is

$$
\Delta \alpha=\operatorname{atan}\left(\frac{\Delta V}{V_{\mathrm{w}}}\right)
$$

On condition that the additional velocity raised by disturbance is far less than the flow velocity, $V \ll V_{\mathrm{w}}$, the equation (17) can be simplified to:

$$
\Delta \alpha \approx \frac{\dot{\alpha} x_{\mathrm{glw}}}{\sqrt{k} V}
$$

The relative increment of the lift is

$$
\Delta L_{\mathrm{w}}=0.5 C_{\mathrm{w}} \rho \sqrt{k} V S_{\mathrm{w}} \dot{\alpha} x_{\mathrm{glw}} .
$$

Then, increment of the pitching torque is

$$
\Delta M_{\mathrm{lw}}=-\left(0.5 C_{\mathrm{w}} \rho \sqrt{k} V S_{\mathrm{w}} x_{\mathrm{glw}}^{2} \cos \alpha\right) \dot{\alpha} .
$$

This torque generated will prevent the pitching motion of the bionic prototype. A new parameter $k_{\mathrm{t}}$ is 
introduced here to give a unified form of the overall damping coefficient of the entire bionic prototype:

$$
\Delta M_{\mathrm{t}}=-k_{\mathrm{t}}\left(0.5 C_{\mathrm{w}} \rho \sqrt{k} V S_{\mathrm{w}} x_{\mathrm{glw}}^{2} \cos \alpha\right) \dot{\alpha},
$$

where value of $k_{\mathrm{t}}$ is usually $1.1 \sim 1.25$ in this kind of calculation.

Depending on equation (19) to equation (21), the overall torque generated by the horizontal tail can be expressed as:

$$
M_{\mathrm{w}}=\Delta M_{\mathrm{lw}}+\Delta M_{\mathrm{t}}
$$

Parameters $k_{\mathrm{t} 1}$ and $k_{\mathrm{t} 2}$ are set as:

$$
\begin{gathered}
k_{\mathrm{t} 1}=0.5 C_{\mathrm{w}} \rho k V^{2} S_{\mathrm{w}} x_{\mathrm{glw}}, \\
k_{\mathrm{t} 2}=0.5 k_{\mathrm{t}} C_{\mathrm{w}} \rho \sqrt{k} V S_{\mathrm{w}} x_{\mathrm{glw}}^{2} .
\end{gathered}
$$

Then the overall torque is

$$
M_{\mathrm{w}}=-k_{\mathrm{t} 1} \cos \alpha \cdot \alpha-k_{\mathrm{t} 2} \cos \alpha \cdot \dot{\alpha}
$$

When the bionic fish swims horizontally and the tail elevator is controlled to swing at angle $\beta$, the additional lift generated by the tail elevator is

$$
\Delta L_{\mathrm{w}}=-0.5 C_{\mathrm{w}} \rho k V^{2} S_{\mathrm{w}} \beta .
$$

The additional torque applied to the gravity center:

$$
\Delta M_{\mathrm{lw}}=\left(0.5 C_{\mathrm{w}} \rho k V^{2} S_{\mathrm{w}} x_{\mathrm{glw}} \cos \alpha\right) \beta=k_{\mathrm{w} 1} \beta .
$$

Through kinematic analysis of the propulsion system by oscillating pectoral foils and considering the angle caused by the disturbance is very small, therefore $\sin \alpha \approx \alpha$ and $\cos \alpha \approx 1$ are obtained. Then:

$$
\begin{gathered}
\left(\mathrm{J}_{\mathrm{b}}+\Delta J_{\mathrm{b}}\right) \ddot{\alpha}+k_{\mathrm{w} 2} \dot{\alpha}+\left(k_{\mathrm{b} 1}-k_{\mathrm{b} 2}+k_{\mathrm{w} 1}\right) \alpha \\
=M_{\mathrm{f}}+\Delta M_{\mathrm{lw}},
\end{gathered}
$$

is obtained, where $J_{\mathrm{b}}$ is the pitching moment of inertia, $\Delta J_{\mathrm{b}}$ is the additional pitching moment of inertia caused by the acceleration of the flow around the bionic robotic fish. Taken the torque generated by the flapping foils as part of disturbance torque, the control equation of pitching movement can be derived as:

$$
\begin{gathered}
\left(\left(J_{\mathrm{b}}+\Delta J_{\mathrm{b}}\right) \ddot{\alpha}+k_{\mathrm{w} 2} \dot{\alpha}+\left(k_{\mathrm{b} 1}-k_{\mathrm{b} 2}+k_{\mathrm{w} 1}\right) \alpha\right) \\
=k_{\mathrm{w} 1} \beta .
\end{gathered}
$$

As can be seen from the above equation, when the horizontal tail swing an angle of $\beta$, it is equal to a step input to a second-order damped pitching system. The system will perform a step response based on this, which is the balance attack angle $\alpha$ relative to the swing angle of horizontal tail when it is in equilibrium state. Therefore, the horizontal tail can be actively controlled to enhance stability of the bionic fish, especially during straight forward swimming. Validity of the control method for enhancing stability through controlling horizontal tail will be verified by simulation in this paper.

\section{SIMULATION MODEL}

It can be observed from the former analysis that a compensating torque of $-k_{\mathrm{w} 1} \beta$ will be generated, when the horizontal tail is controlled to swing a angle of $\beta$. If the torque can be controlled to balance the disturbance torque generated by the flapping pectoral foils, the destabilization affecting swimming performance will be reduced and the stability can be enhanced.

Taking the disturbance torque and the compensating torque by the horizontal tail as an input torque $M$, then after Laplace transform, the following equation can be derived:

$$
\begin{gathered}
\left(J_{\mathrm{b}}+\Delta J_{\mathrm{b}}\right) S^{2} \alpha(s)+k_{\mathrm{w} 2} S \alpha(s) \\
+\left(k_{\mathrm{b} 1}-k_{\mathrm{b} 2}+k_{\mathrm{w} 1}\right) \alpha(s)=M(s) .
\end{gathered}
$$

Then, the transfer function of pitching movement of the bionic fish is:

$$
T(s)=\frac{\alpha(s)}{M(s)}=\frac{1}{J_{\mathrm{eq}} S^{2}+c_{\mathrm{eq}} S+k_{\mathrm{eq}}},
$$

where $J_{\text {eq }}$ is the equivalent moment of inertia, $c_{\text {eq }}$ is the equivalent damping of the system, and $k_{\mathrm{eq}}$ is the equivalent stiffness.

Stability of many underwater devices and ships are controlled by classical proportional-integral-derivative (PID) controller for its simple control architecture (Smallwood and Whitcomb 2004; Sun 2011; Tang et al. 2012). The control architecture for enhancing stability of bionic fish propelled by oscillating paired pectoral fins here is shown in Fig. 4, which is simulated in MATLAB to verify the performance of the control method for compensating stability. The characteristics of the servo used is $\frac{20}{S+20}$. The simulation diagram constructed by Simulink is shown in Fig. 5 .

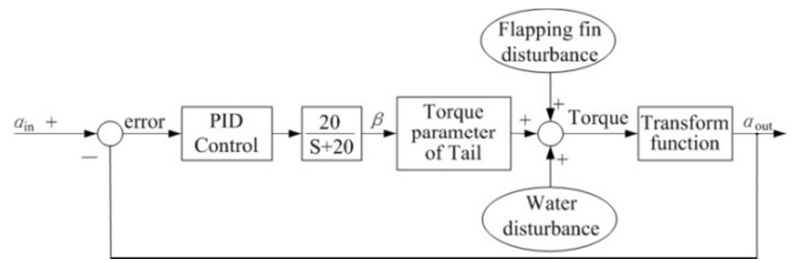

Figures 4: Control Diagram of the Compensating Stability by Horizontal Tail

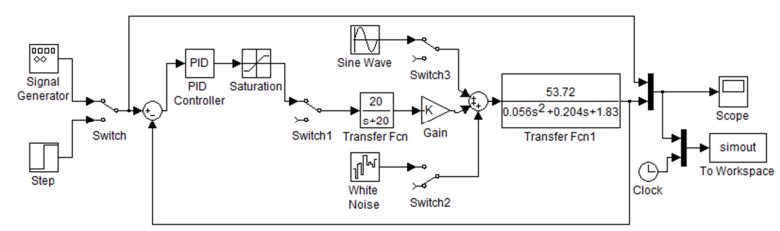

Figures 5: Simulation Diagram of the PID Method of Enhancing Stability 
In the simulation, value of coefficient of the servo, $k$, is 0.98 , value of amplification coefficient of the damping torque $k_{\mathrm{t}}$ is 1.15 , and other parameters are estimated from the model of the bionic robotic fish. Finally, the coefficients in equation (31) can be derived as:

$$
\begin{gathered}
J_{\text {eq }}=0.056, \\
c_{\text {eq }}=0.408 \mathrm{~V}, \\
k_{\text {eq }}=0.64+2.2 \mathrm{~V}^{2} .
\end{gathered}
$$

Results of underwater experiments carried on the bionic fish prototypes have shown that the vertical disturbance of the bionic fish increases as the frequency and amplitude of the flapping movement of the pectoral foils increased. Therefore, if the disturbance amplitude can be controlled to be in an acceptable range in condition of the maximum disturbance caused by the flapping foils, the control method can be applied to other conditions successfully, for the overall disturbance of the bionic fish will be smaller in other conditions.

The maximum flapping frequency of the bionic pectoral foil is $2 \mathrm{~Hz}$, the maximum flapping amplitude is $30^{\circ}$, and the relative swimming velocity is about $0.5 \mathrm{~m} / \mathrm{s}$ in this condition. The transfer function can be calculated based on these values. Variation of the transfer function shown in Fig. 5 is caused by the output is based on degree.

The fuzzy logic control method can mimic control by human to some extent, which is more adaptive to the practical control system (Lee et al. 2012; Wen et al. 2012). The two-dimensional fuzzy controller is introduced to the control system aiming to improving the simulated stability of the bionic fish, which is shown in Fig. 6. The fuzzy controller is constructed by:

(1) The input and output variables: the errors between the actual pitching angle and the desired pitching angle ER, variation of the errors EV are set as the input variables; swing angle of the horizontal tail, $\mathrm{A}$, is set as the output variable.

(2) The fuzzy subset of variables ER, RV and A: minus large, minus middle, minus small, zero, positive small, positive middle and positive large, which can be described by $\{\mathrm{ML}, \mathrm{MM}, \mathrm{MS}, \mathrm{ZE}, \mathrm{PS}, \mathrm{PM}, \mathrm{PL}\}$, the universes of $\mathrm{ER}, \mathrm{EV}$ and $\mathrm{A}$ are $\{-3,-2,-1,0,1,2,3\}$, $\{-3,-2,-1,0,1,2,3\}$ and $\{-4.5,-3,-1.5,0,1.5,3,4.5\}$ respectively. The quantization factors of $\mathrm{EC}$ and $\mathrm{EV}$ are $K_{E C}=1, K_{E V}=1$, and scale factor of the output angle is $K_{\alpha}=5$.

(3) The fuzzy control rule table is constructed based on the following rules: a) If the error of the pitching angle is ML and the error variation EV is also ML, which means that the pitching angle is large and is still becoming larger, then the swing angle of the servo is controlled to be ML; b) If the error of the pitching angle is $\mathrm{ML}$ and the error variation $\mathrm{EV}$ is $\mathrm{PL}$, which means that the pitching angle is large but is becoming small, then the swing angle of the servo is controlled to remain unchanged, ZE actually. C) If the pitching angle is PL and the EV is ML, which means that the pitching angle is large but is becoming small, then the swing angle of the servo is controlled to remain unchanged, $\mathrm{ZE}$ too. Other control rules are constructed with the similar way. The final fuzzy control rule table is shown in Table 1.

TABLE 1: The Fuzzy Control Rule Table

\begin{tabular}{|c|c|c|c|c|c|c|c|}
\hline EV $^{\text {EC }}$ & ML & MM & MS & ZE & PS & PM & PL \\
\hline ML & ML & ML & MM & MM & MS & ZE & ZE \\
\hline MM & ML & MM & MM & MS & MS & ZE & PS \\
\hline MS & MM & MM & MS & MS & ZE & PS & PS \\
\hline ZE & MM & MS & MS & ZE & PS & PS & PM \\
\hline PS & MS & MS & ZE & PS & PS & PM & PM \\
\hline PM & MS & ZE & PS & PS & PM & PM & PL \\
\hline PL & ZE & ZE & PS & PL & PL & PL & PL \\
\hline
\end{tabular}

Based on the fuzzy control parameters, the simulation model is constructed by MATLAB Simulink, as shown in Figure. 6.

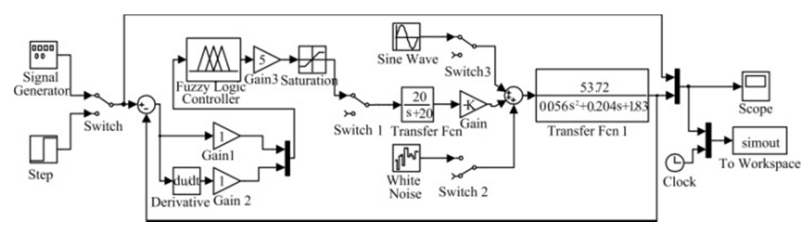

Figures 6: Simulation Diagram of the Fuzzy Logic Method of Enhancing Stability

\section{SIMULATION RESULTS}

Without the compensation of the horizontal tail, variation of the pitching angle of the bionic fish is shown in Fig. 7, when it employs the flapping frequency of $2 \mathrm{~Hz}$ and flapping amplitude of $30^{\circ}$ and there are no effects of incoming flow. As can be observed from the figure, variation frequency of the pitching motion is consistent with the flapping frequency of the pectoral foil, for the variation is mainly caused by the movement of the pectoral foil. The variation amplitude is about $5^{\circ}$ after the state is steady.

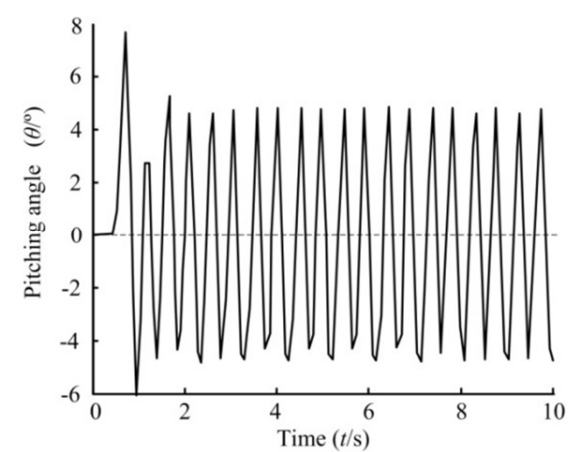

Figures 7: Disturbance without Compensation of the Horizontal Tail 
A better condition of disturbance performance is achieved by compensation movement of the horizontal tail being added, which is shown in Fig. 8. The variation amplitude has been lower to $0.9^{\circ}$, which means that the stability of the bionic fish is improved obviously.

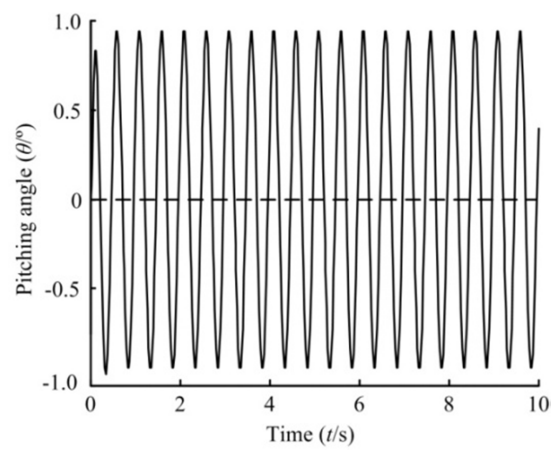

Figures 8: Disturbance with Compensation of the Horizontal Tail

In order to verify the performance of controlling pitching disturbance of the bionic fish by horizontal tail, under condition of flow variation, a random disturbance source is put into the simulation diagram, as shown in Fig. 5. The simulation results are shown in Fig. 9 and Fig. 10. Comparing the results, the compensation effect of the horizontal tail is still working well, as the pitching variation amplitudes are controlled to be approximately within $2^{\circ}$, whereas the simulation results shown in Fig. 9 display a much larger approximate vibration range of $10^{\circ}$ without performance of horizontal tail.

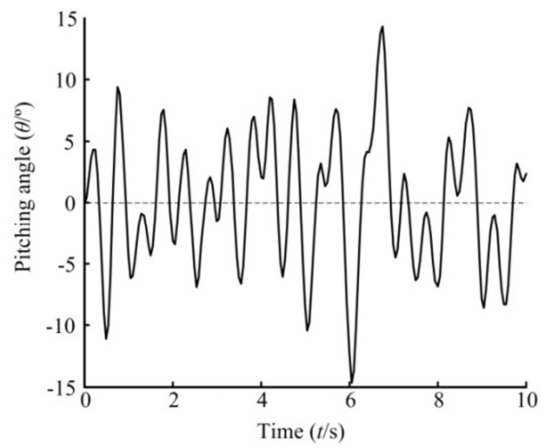

Figures 9: Disturbance without Compensation of the Horizontal Tail under Water Flow

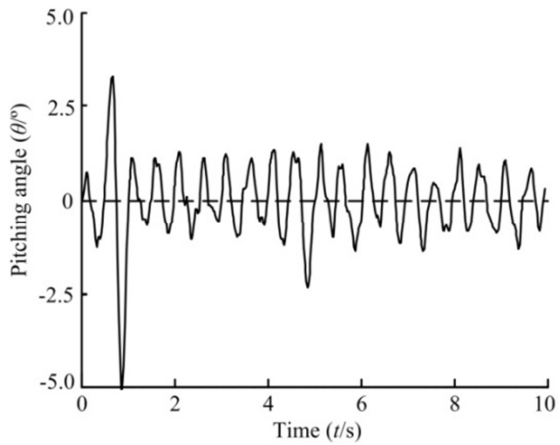

Figures 10. Disturbance with Compensation of the Horizontal Tail under Water Flow
A better stability performance has been obtained by introducing the fuzzy logic control method, as shown in Fig. 11. Same parameters of the structure and movement of the bionic fish are used in simulation of fuzzy logic control method. The disturbance pitching angles are constricted within $-0.5^{\circ}$ to $0.5^{\circ}$, comparing with the relative larger disturbance angle ranges achieved by the PID method shown in Fig. 10.

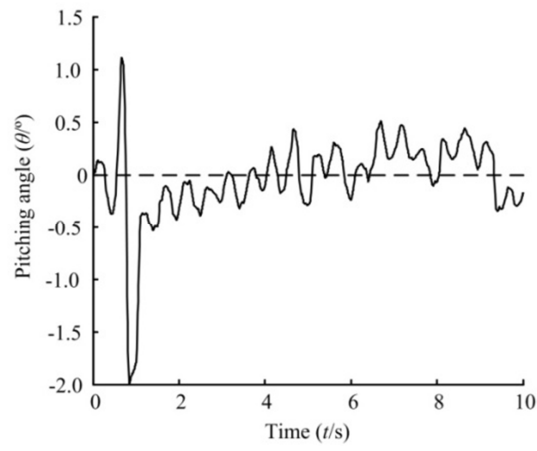

Figures 11. Disturbance with Compensation of the Horizontal Tail Utilizing Fuzzy Control Method

\section{CONCLUSION}

Researchers have been focused on developing prototypes of bionic fish propelled by oscillating paired pectoral foils to possess attractive features of their natural samples, such as high speed, high efficiency and low noise. The stability is one of the important factors to realizing these objectives. The main causes of disturbance are raised by variation of the lift force generated by the flapping pectoral foils, which cannot be reduced obviously by optimization of the structures and movement characteristics of the bionic fish, for the object of keeping the bionic fish with desired swimming velocity. Therefore, the method of enhancing stability by auxiliary work of the horizontal tail that is mounted for realizing movements of up-floating and diving. The PID method and fuzzy logic control method are introduced in the simulations of control performances. The simulation models are constructed by Simulink and parameters utilized in the simulations are determined by characteristics of the bionic fish, including characteristics of the servos used. A white noise is added to the simulation models to mimic variation of the incoming flow.

Simulation results show that with auxiliary work of the horizontal tail, an improved performance of pitching disturbance can be obtained. The disturbance pitching angle can be controlled within a range of $-1^{\circ}$ to $1^{\circ}$ without influence of incoming flow with auxiliary work of horizontal tail. The variation of the incoming flow has great effect on stability performance of the bionic fish, for a wide range of pitching angle of $-10^{\circ}$ to $10^{\circ}$ in stable state has been observed in condition of a white noise being introduced even with adjusting compensation of the horizontal tail. Whereas, controlling the swimming motion of the horizontal tail 
by fuzzy logic method provides a way to solve this problem. The fuzzy control rules are constructed based on the common two-dimensional controller. The disturbance pitching angles can be restricted within a range of $-0.5^{\circ}$ to $0.5^{\circ}$ even encountered incoming flow, actually a white noise in the simulation model.

The PID control method and the fuzzy logic control method presented in this paper will be further applied to the bionic fish prototype to verify its practicality and improved based on the experimental results.

\section{ACKNOWLEDGEMENT}

The work presented in this paper was partly supported by the National Natural Science Foundation of China (No. 51205011), the Chinese Postdoctoral Science Foundation (No. 2012M510304) and the Research Fund for the Doctoral Program of Higher Education of China (No. 20101102110022). Thanks are also due to other members in bionic fish research team graduated or not, for their help and support to the presented work.

\section{REFERENCES}

Anderson J M and Chhabra N K. 2002. "Maneuvering and stability performance of a robotic tuna," Integrative and Comparative Biology, vol. 42, pp. 118-126.

Cai Y R, Bi S S and Zheng L C. 2010. "Design and experiments of a robotic fish imitating cow-nosed ray," Journal of Bionic Engineering, vol. 7, pp. 120-126.

Chen Z, Um T I, Zhu J Z and H Bart-Smith. 2011. "Bio-inspired robotic cownose ray propelled by electroactive polymer pectoral fin," Proceedings of the ASME 2011 International Mechanical Engineering Congress \& Exposition (IMECE 2011), pp. 1-8.

Cai Y R, Bi S S and Zheng L C. 2012. "Design optimization of a bionic fish with multi-joint fin rays," Advanced Robotics, vol. 26, pp. 177-196.

Elizabeth P. 2011. "Bio-inspired engineering: Manta machines Science," vol. 232, pp. 1028-1029.

Fang Z P. 2005. "Aircraft dynamics", Beijing: Beihang University Press.

Harris J E. 1938. "The role of the fins in the equilibrium of the swimming fish II: The role of the pelvic fins," Journal of Experimental Biology, vol. 13, pp. 476-493.

$\mathrm{Hu} \mathrm{T}$ J, Wang G M, Shen L C and Li F. 2006. "Bionic inspirations of fish-like robots from Rhinecanthus Aculeatus," Proceedings of the 2006 IEEE International Conference on Mechatronics and Automation, pp. 639-643, 25-28, June.

IMAE, 2012. Available at: http://www.imae-kagaku.com/m ech.htm (in Japanese), accessed in November.

Lee P J, Lee M S and Wang R C. 2012. "A fuzzy control based robotic fish with multiple actuators," International Journal of Fuzzy Systems, vol. 14, pp. 45-53.

Parson J M, Fish F E and Nicastro A J. 2011. "Turning performance of batoids: Limitations of a rigid body," Journal of Experimental Marine Biology and Ecology, vol. 402, pp. 12-18.

Rosenberger L J. 2001. "Pectoral fin locomotion in batoid fishes: Undulation versus occillation," The Journal of Experimental Biology 204, pp. 379-394.
Smallwood D A and Whitcomb L L. 2004. "Model-based dynamic positioning of underwater robotic vehicles: theory and experiment," IEEE Journal of Oceanic Engineering, vol. 29, pp. 169-186.

Suzuki H, Kato N, Suzumori K. 2007. "Load characteristics of mechanical pectoral fin," Experiments in Fluids, vol. 44, pp. 759-771.

Sun Y Q. 2011. "Modern maneuver theory and application of submarine and deep-diving submersible vehicle," Beijing: National Defense Industry Press.

Sefati S, Neveln I, Maciver M A, et al. 2012 "Counter-propagating waves enhance maneuverability and stability: A bio-inspired strategy for robotic ribbon-fin propulsion," The 4th IEEE RAS\&EMBS International Conference on Biomedical Robotics and Biomechatronics (BioRob), pp. 1620-1625, 24-27, June.

Tang Z J, He Q B, Wang S A, et al. 2012. "An improved generalized predictive control for AUV yaw," Advanced Materials Research, pp. 1709-1713.

Webb P W. 2002. "Kinematics of plaice, Pleuronectes platessa, and cod, Gadus morhua, swimming near the bottom," Journal of Experimental Biology, vol. 205, pp. 2125-2134

Wang T M, Liang J H, Shen G X, et al. 2005. "Stabilization based design and experimental research of a fish robot," IEEE/RSJ International Conference on Intelligent Robots and Systems, pp. 954-959, 2-6 August.

Wang Z L, Wang Y W, Li J, et al. 2009. "A micro biomimetic manta ray robot fish actuated by SMA," Proceedings of the 2009 IEEE International Conference on Robotics and Biomimetics, pp. 1809-1813.

Wilson M M and Eldredge J D. 2011. "Performance improvement through passive mechanics in jellyfish-inspired swimming," International Journal of Non-linear Mechanics, vol. 46, pp. 557-567.

Wen L, Wang T M, Wu G H, et al. 2012. "Novel method for the modeling and control investigation of efficient swimming for robotic fish," IEEE Transactions on Industrial Electronics, vol. 59, pp. 3176-3188.

Xu Y C, Zong G H, Bi S S and Gao J. 2007. "Initial development of a flapping propelled unmanned underwater vehicle (UUV)," Proceedings of 2007 IEEE International Conference on Robotics and Biomimetics, pp. 524-529.

Xu Y C, Zong G H, Bi S S, et al. 2012. "Pitching stability analysis of the pectoral fin propelled robot fish," Robot, vol. 34, pp. 375-379.

Yu J Z, Su Z S, Wang M, et al. 2012. "Control of yaw and pitch maneuvers of a multilink dolphin robot," IEEE Transactions on Robotics, vol. 28, pp. 318-329.

Zhou C L and Low K H. 2010. "Better Endurance and Load capacity: An Improved Design of Manta Ray Robot (RoMan-II)," Journal of Bionic Engineering, vol. 7, pp. S137-S144. 